(C) 1993 IEEE. Reprinted from IEEE Transactions on Rehabilitation Engineering, 1(2).

This material is posted here with permission of the IEEE. Such permission of the IEEE does not in any way imply IEEE endorsement of any of The University of Melbourne's products or services. Internal or personal use of this material is permitted. However, permission to reprint/republish this material for advertising or promotional purposes or for creating new collective works for resale or redistribution must be obtained from the IEEE by writing to pubs-permissions@ieee.org.

By choosing to view this document, you agree to all provisions of the copyright laws protecting it. 


\title{
A Portable Programmable Digital Sound Processor for Cochlear Implant Research
}

\author{
Hugh J. McDermott, Andrew E. Vandali, Richard J. M. van Hoesel, \\ Colette M. McKay, J. Mark Harrison, and Lawrence T. Cohen
}

\begin{abstract}
A programmable sound processor which utilizes dig ital signal processing has been developed for hearing prosthesis research. It incorporates a Motorola DSP56001 integrated circuit, 32K words of memory, a 12 b analog-to-digital converter, and a data formatter and transmitter which conveys control codes to the receiver-stimulator of a cochlear implant. The processor is pocket-sized and battery powered. It has been programmed to emulate the Spectral Maxima Sound Processor for the University of Melbourne/Nucleus 22 electrode implant, and is currently being used by several implantees. In continuing research, speech processing programs are being improved, and other applications, including signal processing for binaural implants and advanced hearing aids, are being developed.
\end{abstract}

\section{INTRODUCTION}

$\mathbf{T}$ HE use of cochlear implants to restore limited auditory capabilities to both adults and children with profound to total sensorineural hearing loss is now an established and widely accepted procedure. These devices create hearing sensations by electrical stimulation of residual auditory neurons. The cochlear implant presently manufactured by Cochlear Pty Limited, based on research undertaken by the University of Melbourne [1], stimulates by means of an array of 22 electrodes which is placed in the scala tympani of the cochlea. An implanted receiver-stimulator delivers biphasic current pulses to selected pairs of electrodes. The receiver-stimulator receives data specifying the parameters of the stimuli as well as power from an external speech processor via a transcutaneous inductive coupling. In general, speech processors are designed to modify or analyze sound signals obtained from a microphone and to encode selected components of those signals for transmission to the receiverstimulator. It is now practical to process speech signals digitally in real-time using portable, battery-powered hardware [2], [3]. Development of better speech processing techniques has enabled, and probably will continue to enable, improvements in the perceptual performance of cochlear implantees.

The first successful speech processor developed for the 22 electrode implant extracted and presented information about the fundamental frequency $(F 0)$ and second formant $(F 2)$ of speech. The fundamental frequency was used to control

\footnotetext{
Manuscript received October 8, 1992; revised July 27, 1993. This work was supported by the NIH under Contract N01-DC-9-2400, the National Health and Medical Research Council of Australia, the Australian Research Council, and the Australian Bionic Ear and Hearing Research Institute.

The authors are with the Australian Bionic Ear and Hearing Research Institute, East Melbourne, Victoria 3002, Australia.

IEEE Log Number 9212543.
}

the stimulation pulse rate whenever voicing was detected, while the $F 2$ frequency determined which electrode was activated. The amplitude of the second formant controlled the current level of the stimuli. These parameters were selected because they were considered most likely to assist implantees in understanding speech, and because they matched some characteristics of electrically induced hearing effectively. Psychophysical studies had shown that two distinct aspects of pitch could be perceived, one depending on the rate of stimulation (up to approximately $300 \mathrm{~Hz}$ ), and the other on the location of stimulation within the cochlea. Loudness was found to depend on the current level [4].

As this processing technique, known as the $F 0 / F 2$ strategy, provided considerable benefit to most implantees [5], [6], further research led to the inclusion of information about the first formant $(F 1)$. The additional information was presented by activating a second electrode nonsimultaneously within each stimulus period, at a location determined by the $F 1$ frequency. Users of the WSP III processor implementing this $F 0 / F 1 / F 2$ strategy obtained generally improved speech perception [7], [8].

Subsequently, the Mini Speech Processor (MSP) was developed by Cochlear Pty Limited. It utilizes digital signal processing techniques and can be programmed to implement a variety of speech processing strategies. The most advanced strategy currently available commercially, known as Multipeak, is an extension of the $F 0 / F 1 / F 2$ strategy which activates four electrodes within each stimulus period. The two additional stimuli convey information derived from three higher frequency bands in a range above 2 $\mathrm{kHz}$, and are delivered to fixed electrodes towards the basal end of the cochlea. The MSP, programmed with Multipeak, is providing better performance to implantees than its predecessors, particularly in the presence of competing noise [9].

More recently, an advanced sound processor based on programmable digital signal processing has been designed in the University of Melbourne for use with the 22 electrode implant. The processor, which henceforth will be referred to as the P-DSP, was first provided to a number of implantees late in 1991 so that innovative speech processing strategies could be evaluated. The first strategy implemented was a version of the Spectral Maxima Sound Processor (SMSP) [10], which was developed by the University in 1989, and implemented originally using analog signal processing circuitry. The current design of the SMSP is discussed in detail in Section III below. 


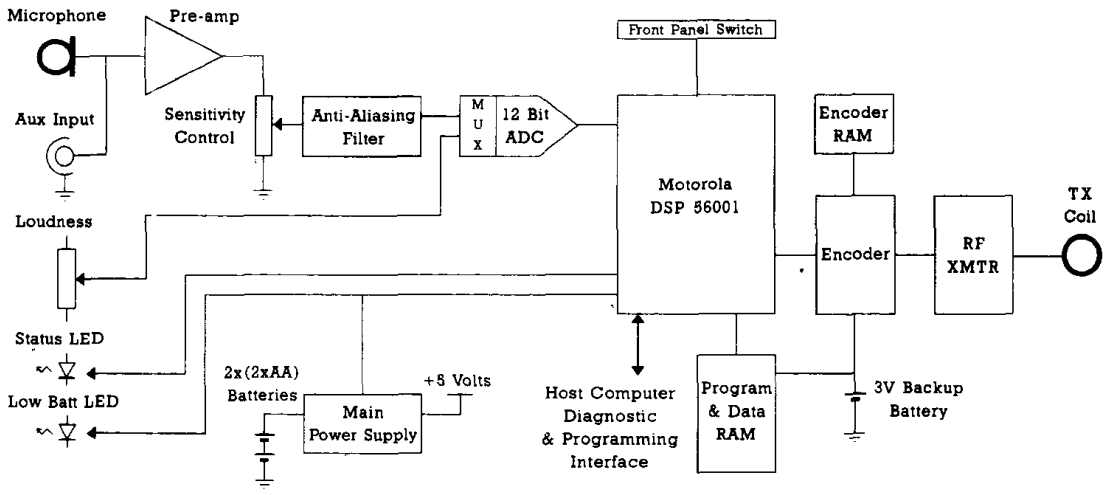

Fig. 1. Block diagram showing the main functional units and front-panel controls of the P-DSP.

In assessing speech processing techniques, it is important that subjects are able to gain experience using the processor in everyday conditions away from the laboratory. The design of the P-DSP was directed towards this end. It is pocket-sized, with dimensions of $136 \times 73 \times 23 \mathrm{~mm}$, and a complete unit including four batteries weighs $286 \mathrm{~g}$. The batteries, which are AA size, may be either alkaline or rechargeable types. When running a typical speech processing program, a set of alkaline batteries provides about $30 \mathrm{~h}$ of continuous operation.

\section{HARDWARE DESIGN}

The main objective guiding the development of the $\mathrm{P}$ DSP was the need for flexibility to facilitate the design and modification of speech processing programs for research. As shown in the block diagram of Fig. 1, the core of the system is a digital signal processing integrated circuit (Motorola DSP56001) and memory which stores both program code and data. Random-access memory (RAM) is used so that programs can be loaded rapidly from a host computer. Data retention while the unit is switched off is ensured by means of a small lithium back-up battery.

In a typical application, the system works in the following way. Signals picked up by the microphone are preamplified with gain determined by a front-panel sensitivity control. They then pass to an analog-to-digital converter (ADC) via an antialiasing filter, which is a 6th-order Butterworth design with a cutoff frequency of $6 \mathrm{kHz}$. A deliberate decision was made to permit aliasing of signals at frequencies just above $6 \mathrm{kHz}$ for reasons that are explained later. The ADC (Linear Technology LTC1290) samples the filtered signals at a rate of 12000 samples/s, and conveys $12 \mathrm{~b}$ values to the DSP via a serial interface. The DSP, running at a clock frequency of typically $6 \mathrm{MHz}$, repeatedly executes the program stored in the RAM, which has a capacity of 32768 words. (Each word contains 24 b.)

The main output of the DSP is via a parallel interface to a programmable logic device (Xilinx XC3000 series). This device is configured to arrange sets of data in the format required by the receiver-stimulator of the implant, and is known as the encoder. Its logic configuration is defined by data stored internally and maintained by the back-up battery when required. The encoder constructs frames in a special format using data stored temporarily in an associated RAM, and controls a radio frequency (RF) transmitter. The durations of RF bursts within a frame specify the active electrodes, current level and pulse width of a single stimulus pulse to be generated by the receiver-stimulator. When sounds are being processed by the P-DSP, appropriate data frames are transmitted to the implant periodically.

The P-DSP's battery power supply is based on a switching converter which can provide a regulated $5 \mathrm{~V}$ output at load currents of over $100 \mathrm{~mA}$ with individual cell voltages between 0.8 and $1.6 \mathrm{~V}$. The efficiency of the converter is approximately $85 \%$. All circuits in the P-DSP are powered by the $5 \mathrm{~V}$ supply. When the battery voltage is too low to ensure correct system operation, a signal which prevents transmission of data to the implant is sent to both the encoder and the DSP.

In addition to the sensitivity control, a general purpose potentiometer is provided on the front panel of the P-DSP. The setting of this potentiometer is measured periodically by the ADC, which incorporates an 8 channel analog multiplexer at its input. A typical use of the potentiometer is as a loudness control so that implantees can adjust the overall loudness of sounds independently of the microphone's sensitivity. The front panel also provides a $3.5 \mathrm{~mm}$ socket for connection of various alternative audio signal sources, such as telephone or television adapters.

The main power switch has four positions, three of which are connected to an input port of the DSP. When appropriate software is executing, P-DSP users can choose from up to three different programs or functions. Two light-emitting diodes provide users with information about the functional status of the P-DSP. One is illuminated when the batteries are becoming flat as a warning that they need to be replaced or recharged. The illumination of the other is controlled by the DSP program. It may be used, for example, to indicate that an input signal is present, that a particular program option has been enabled, or that an internal fault has been detected.

Normally implantees use the P-DSP with the microphone headset supplied by Cochlear Pty Limited for use with their 
SPECTROGRAM

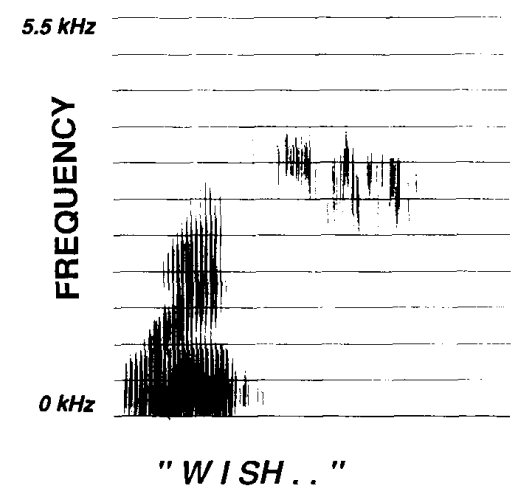

STIMULI

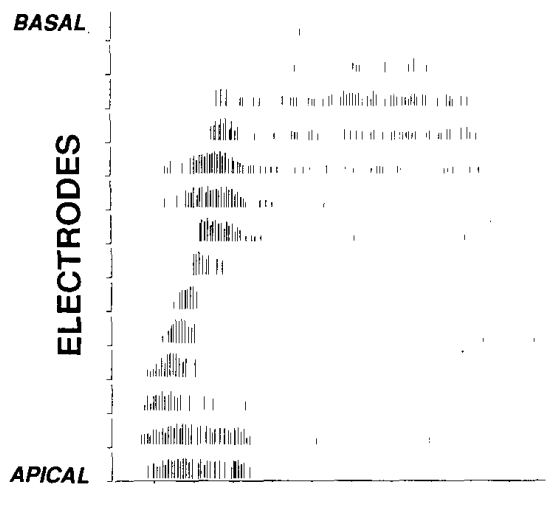

Fig. 2. Stimulation patterns (right panel) produced by the SMSP when processing the spoken word "wish", whose spectrogram appears in the left panel. Each vertical line segment represents one pulse of electrical stimulation. The horizontal axis represents time; the overall duration of the word is approximately $0.65 \mathrm{~s}$.

Mini Speech Processor (MSP) [3]. The headset contains a miniature directional microphone worn on the pinna and a transmitting coil. The transmitting coil is held over the receiving coil of the receiver-stimulator by a permanent magnet which is aligned with a matching magnet mounted on the implant package. The headset is connected to the processor by a lightweight flexible cable.

The electronic components of the P-DSP are mounted on two printed circuit boards. In order to minimize internally generated electrical noise and interference, the boards have multiple layers and incorporate ground planes. The analog signal processing components are mostly located on one board while the other contains the digital components. The crystal that determines the clock frequency of the DSP can be changed easily so that a frequency can be selected which minimizes the power consumption for the required level of computational performance.

Software for the P-DSP is developed on a host computer (IBM PC or compatible) which is fitted with a specially designed interface card. The interface can be used to load, monitor and control programs, and to examine or modify memory contents even while the P-DSP is executing speech processing programs.

\section{P-DSP SOFTWARE: THE SMSP}

As an example of the practical operation of the P-DSP for cochlear implantees, the emulation of the speech processor called the Spectral Maxima Sound Processor (SMSP) is described below. Since the original (analog) version of the SMSP [10] was shown to enable better speech understanding performance than the MSP (programmed with the Multipeak strategy) for a small group of implantees [11], [12], a version employing digital signal processing techniques was developed for the P-DSP. Functionally this version is essentially identical to the original SMSP, and generates very similar patterns of electrical stimulation. The digital implementation does provide some technical advantages, such as significantly increased dynamic range.

The principle of operation of the SMSP can be summarized as follows: incoming sound signals are divided into a number of frequency bands; a subset of those bands having the highest amplitudes is selected periodically; and corresponding electrode pairs in the user's implant are activated at levels related to the amplitudes. Note that the term maxima here denotes the largest spectral estimates, not the local peaks in the spectrum. Typically, several maxima are selected in the vicinity of a single spectral peak. The present implementation of the SMSP has 16 bandpass filters which are allocated in tonotopic order to 16 electrode pairs. Normally the electrodes used are the 16 most-apical that are available on the 22 electrode array. The six filters with the highest amplitudes are selected at intervals of $4 \mathrm{~ms}$, resulting in a constant rate of stimulation. The levels of the stimulus pulses are related to the filter output amplitudes by a function which is approximately logarithmic.

Referring to Fig. 2, the right panel illustrates the pattern of stimulation produced by the SMSP when processing the spoken word "wish". The length of each vertical line segment represents the current level of each pulse of stimulation generated by the cochlear implant. Comparison with the spectrogram on the left shows that the SMSP activates electrodes at positions corresponding to the first and second formant frequencies during the vowel, and stimulates on several basal electrodes during the fricative consonant. This is achieved without any direct attempt at extracting speech features such as the formant frequencies. By contrast with the MSP, which activates one electrode for each of $F 1$ and $F 2$, the SMSP often activates two or more nearby electrodes to represent these spectral features, as is evident in Fig. 2 for the interval when the $F 2$ frequency is rising. This may convey more information to implantees about the centre frequencies and bandwidths of speech formants. The simple technique. of selecting the largest spectral components is also less affected by competing noise than the formant frequency estimation algorithms of the MSP, and enables the SMSP to provide substantial benefit 


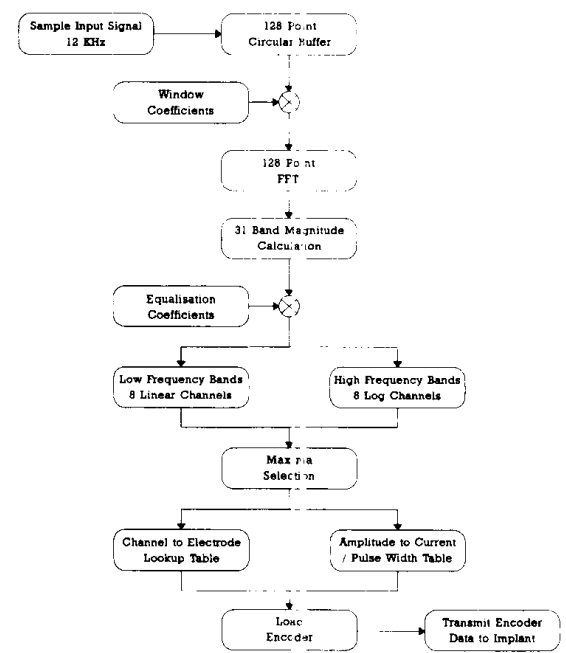

Fig. 3. Signal processing in the P-DSP emulating the Spectral Maxima Sound Processor.

to implantees in noisy conditions [11]. Another difference between the SMSP and MSP is that the SMSP stimulates at a constant rate and does not attempt to detect voicing or estimate the fundamental frequency of speech. Instead, fundamental frequency information is probably conveyed in the periodic variations of the stimulus amplitude envelope, especially during steady vowels.

The operation of the P-DSP version of the SMSP is outlined schematically in Fig. 3. Input signals sampled by the ADC are placed in a circular buffer that can store 128 samples. The ADC interrupts the DSP as soon as each sample is available. Note that unlike the original version of the SMSP, the P. DSP does not compress signals before they undergo spectral analysis. The higher dynamic range available in the digital processor has obviated the need for input compression.

The samples are windowed to produce a frequency response for the spectral analysis comparable with that of the original SMSP. In that design, an analog filterbank chip provided 16 frequency channels, each comprising a bandpass filter, rectifier and low-pass filter. The lowpass filters were designed to smooth the amplitude estimates in time, and were set to a cutoff frequency of $200 \mathrm{~Hz}$. In the P-DSP an equivalent function was provided by appropriate design of the window. The shape of the window in the frequency domain resembles that of the Daniall window [13], which is specified in terms of its Fourier transform by

$$
\begin{aligned}
W_{j / 2} & =(2 m+1)^{-1}, \text { for } j=-m,(-m+1), \cdots,(m-1), m ; \\
& =0 \text { otherwise; }
\end{aligned}
$$

where $m$ is an integer constant. The gradient of the transition band was reduced and the corresponding coefficients in the time domain were then smoothed by a Kaiser window [14] to eliminate discontinuities. This produced the desired frequency response with greater than $50 \mathrm{~dB}$ attenuation of the sidelobes. The final window coefficients are shown graphically in Fig. 4(a), and the resulting frequency response is shown in Fig. 4(b).

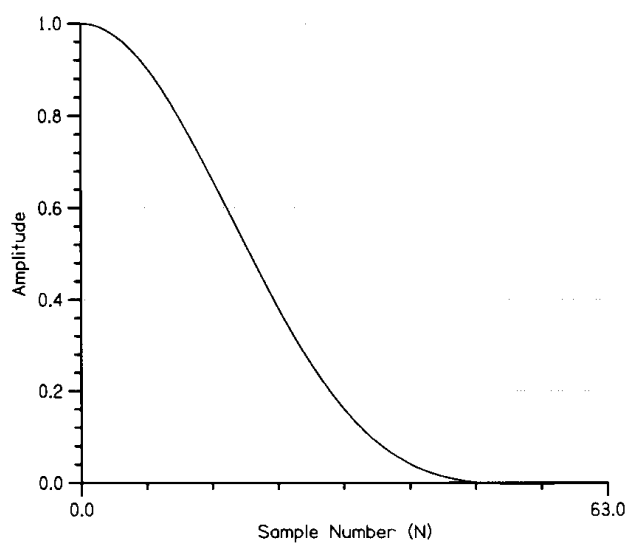

(a)

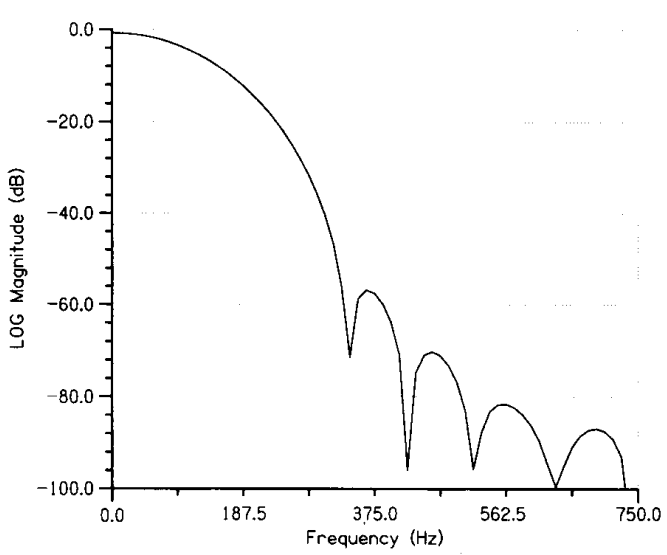

(b)

Fig. 4. Window design for the SMSP strategy. The coefficients are plotted graphically in (a) with the corresponding frequency response down to $100 \mathrm{~dB}$ attenuation in (b). In each case half of the function is omitted for clarity.

A fast Fourier transform (FFT) is performed on the windowed data, resulting in 65 spectral amplitude estimates spaced equally in frequency from 0 to $6 \mathrm{kHz}$. As a consequence of the window shape, the effective $-3 \mathrm{~dB}$ bandwidth of each FFT component is $187.5 \mathrm{~Hz}$, which equals twice their spacing. Since the spectrum is thus completely represented by the oddnumbered FFT components, the even-numbered components are omitted from subsequent processing. The component at $93.75 \mathrm{~Hz}$ is also ignored, as it is below the range of frequencies encompassed by the original analog SMSP. This leaves a total of 31 spectral magnitude estimates, which are scaled by a set of values to shape the overall frequency response of the processor and microphone. As in the original version of the SMSP, the values are chosen so that the sensitivity of the complete system approximates that of normal hearing at a loudness level of 70 phon.

Next the spectral estimation is reduced to a set of 16 frequency components which can be allocated to the stimulating electrodes. The lower part of the spectrum is estimated using eight linearly spaced bands from 375 to $1687.5 \mathrm{~Hz}$. At higher frequencies, eight bands of greater bandwidth are 
TABLE I

Channel Frequencies for SMSP Spectral ANalysis

\begin{tabular}{ccc}
\hline Band & \multicolumn{2}{c}{ Center Frequency (Hz) } \\
\cline { 2 - 3 } & Analog SMSP & Digital SMSP (P-DSP) \\
\hline 1 & 250 & 375 \\
2 & 450 & 562 \\
3 & 650 & 750 \\
4 & 850 & 937 \\
5 & 1050 & 1125 \\
6 & 1250 & 1312 \\
7 & 1450 & 1500 \\
8 & 1650 & 1687 \\
9 & 1900 & 1969 \\
10 & 2150 & 2344 \\
11 & 2500 & 2719 \\
12 & 2900 & 3094 \\
13 & 3300 & 3562 \\
14 & 3800 & 4125 \\
15 & 4500 & 4687 \\
16 & 5400 & 5531 \\
\hline
\end{tabular}

created by combining contiguous subsets of the remaining FFT components. As shown in Table I, the resulting spectral analysis comprises 16 bands whose centre frequencies are comparable with those used in the original SMSP design. Some extension of the P-DSP's frequency response results from aliasing of signals at frequencies between $6 \mathrm{kHz}$ and approximately $7.7 \mathrm{kHz}$ which are not totally eliminated by the anti-aliasing filter. Such signals will appear in the two highest spectral bands. In speech, this frequency region is dominated by fricatives, some of which can have most energy above $6 \mathrm{kHz}$. For good speech comprehension it is importan to indicate the presence of these sounds. Moreover, as they are characterized by a broad distribution of energy, the loss of precise frequency information is probably not a serious disadvantage.

The 16 values are then sorted into order of decreasing amplitude. The six largest are selected, and the corresponding bands determine which electrodes are to be activated. The allocation of electrodes to spectral bands is specified in a lookup table in RAM so that it may be altered easily for different implantees. The amplitudes are converted into levels of stimulation via a second lookup table which normally contains an approximately logarithmic transfer function. The values contained in this table are constrained to lie between the maximum comfortable loudness level and the threshold level (at which sensations are just audible) for each electrode. The ratio of these two levels varies considerably across electrodes and among implantees, but is usually less than $20 \mathrm{~dB}$. As the amplitude estimates obtained from the analysis of incoming signals encompass a much larger dynamic range (up to 46 $\mathrm{dB}$ in the P-DSP), the lookup table is needed to convert the amplitudes to suitable levels for each electrode.

The loudness control also affects the range of output levels produced. When sounds are being processed with the loudness control set below its upper limit, some stimuli may be generated at levels below the nominal threshold level. However, there are no circumstances under which levels can be generated above maximum comfortable loudness. In practice, SMSP users typically set the loudness control to maximum except in unusually noisy conditions.
The levels of stimulation may be controlled either by varying the current levels with fixed pulse widths, or by varying both the currents and pulse widths together. A third lookup table specifies the conversions required in each case. This method of mapping spectral amplitudes onto stimulus levels follows closely the technique incorporated in the MSP speech processor [9]. As most SMSP users have had considerable experience with the MSP, it was preferable to avoid unnecessary changes in the processing algorithm which could affect the sound quality and cause difficulties to implantees during the change-over period.

In the final phase of the processing algorithm, data specifying the level, pulse width and active electrodes for each stimulus pulse to be generated are transferred to the encoder. If fewer than six of the 16 bands are found to have significant amplitudes, then correspondingly fewer stimuli will be generated. While the encoder formats the data and controls the RF transmitter, the DSP is able to continue processing simultaneously. After a delay which determines the overall rate of stimulation, the DSP repeats execution of the entire algorithm. Rates up to $400 \mathrm{~Hz}$ for the group of six pulses are practical with the P-DSP version of the SMSP, which is significantly higher than was possible with the original SMSP. Because of the time required to transmit the RF data frames to the implant, higher rates usually cannot be achieved unless the implantee's comfortable levels of stimulation can be attained using exceptionally small pulse widths.

As mentioned earlier, the power switch of the P-DSP has four positions. Currently their functions are 1) off, 2) test, which generates a continuous pulse train at a comfortable level to verify system function and to assist with transmitting coil alignment, 3) normal, which executes the speech processing algorithm just described, and 4) special, which is similar to 3) but attempts to reduce background noise by suppressing stimulation when input signal amplitudes fall below a predetermined value. Selecting function (4) effectively removes approximately $12 \mathrm{~dB}$ from the P-DSP's input dynamic range by modifying the lower third of the amplitude to stimulus level lookup table. The transfer function in the upper twothirds of the table follows the logarithmic function described above which is used when the switch is on position 3 ). Implantees who are annoyed by continuous low levels of electrical stimulation frequently choose switch position 4) when listening in noisy environments.

\section{PERFormanCE OF IMPLANTEES}

The speech perceptual performance of a small group of implantees using the original version of the SMSP has been compared to that obtained using the MSP (programmed with the Multipeak strategy) [11], [12]. Tests were carried out using sound only at a level of 65-70 dBA. Substantial improvements were achieved with the SMSP for recognition of closed-set vowels and consonants and for comprehension of open-set monosyllabic words. Furthermore, understanding of openset sentences both in quiet and in competing noise was significantly improved. 
More recently, four of the implantees who were experienced with the original SMSP have become users of the P-DSP. They have all commented on the newer processor's improved clarity and reduced levels of background noise. However, they also report that the overall sound quality of the two SMSP implementations is very similar. In order to verify that the PDSP is providing these implantees with at least their previous levels of performance, speech perception data collected around the time of the changeover were examined. Results from three monosyllabic word tests for each subject using each processor were averaged. The data were collected for the last three test sessions using the original version of the SMSP and for the first three sessions using the P-DSP programmed with the SMSP strategy. In each test a recorded list of 50 words, each word having a consonant-vowel-consonant structure [15], was presented. The lists were phonemically balanced and each subject was tested with a different list in each of their six sessions.

The results are shown in Fig. 5. The scores include percentages correct of the vowels and consonants within the words as well as the percentages correct of the whole words. When using the P-DSP, all subjects performed as well as or better than when using the original (analog) version of the SMSP. These results need to be interpreted with caution, however, as in the case of Subjects 3 and 5 a period of several months' duration, during which alternative speech processors were being evaluated, intervened between the two groups of test sessions. Furthermore, although each subject had had at least two months' experience with the SMSP strategy before changing processors, it is possible that increases in scores might be due partly to ongoing learning. Nevertheless, the test results combined with the implantees' comments indicate that the performance of the P-DSP is at least equal to, and probably better than, that of the original version of the SMSP. This assertion is supported by the fact that the patterns of stimulation produced by the two versions of the SMSP processing the same speech items appear very similar when examined visually. Evaluation of P-DSP users' speech comprehension abilities with the SMSP and other processing algorithms is continuing [16].

\section{CONCLUSION}

A programmable sound processor utilizing digital signal processing techniques has been developed. Designed particularly for use with a cochlear implant hearing prosthesis, it is pocket-sized and battery powered. A photograph of a complete unit appears in Fig. 6. The Spectral Maxima Sound Processor, which has recently been shown to provide improved speech recognition performance for implantees, has been implemented successfully on the P-DSP.

As the P-DSP facilitates modifications to the structure of processing schemes, or to the values of the parameters which control them, it is an ideal tool for cochlear implant research. It is suitable also for research into related speech processing applications. There is provision for a second encoder and RF transmitter so that the P-DSP can be used with subjects implanted bilaterally. There is also provision for a second microphone and analog preprocessor so that two acoustic

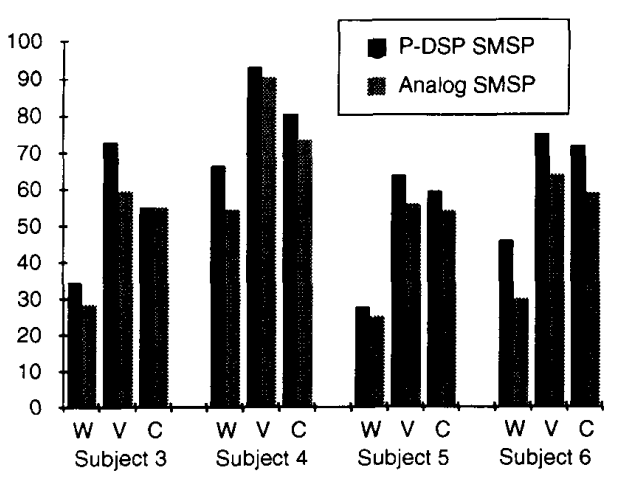

Fig. 5. Recognition of monosyllabic words by four cochlear implantees using the SMSP strategy. Percent correct scores are shown for the original SMSP and for the P-DSP version. Each value plotted represents the average result for three different lists of words. Results for whole words correct (W) are shown, alongside the results for the vowels (V) and consonants (C) within the words.

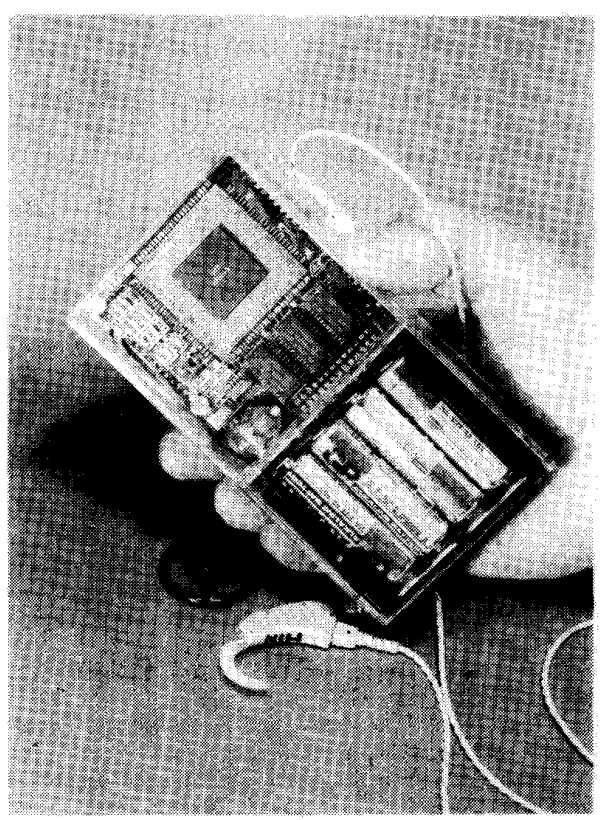

Fig. 6. Photograph of the P-DSP processor showing the internal electronics, batteries, and externally worn headset.

signals can be digitized and processed concurrently. With modifications, a digital-to-analog converter and amplifier can be incorporated into the P-DSP so that it can be used in advanced hearing aid research. Another possibility is to process sounds for a cochlear implant and a hearing aid simultaneously. Presently, several of these applications are being developed and evaluated with hearing impaired volunteers.

\section{ACKNOWLEDGMENT}

Many people have contributed to the development of the P-DSP, especially our colleagues Dr. Y. C. Tong and Prof. G. M. Clark. Mr. M. Robinson assisted with the engineering. We would also like to thank the cochlear implantees who have willingly taken part in the research. 


\section{REFERENCES}

[1] G. M. Clark, The University of Melboume--Nucleus Multi-Electrode Cochlear Implant, Advances in Oto-Rhino-Laryngology. Basel: Karger, 1987, vol. 38.

[2] L. R. Morris and P. Barszczewski, "Algorithms, hardware and software for a digital signal processor microcomputer-based speech processor in a multielectrode cochlear implant system," IEEE Trans. Biomed. Eng., vol. 36, pp. 573-584, June 1989 .

[3] J. F. Patrick, P. M. Seligman. D. K. Money, and J. A. Kuzma "Engineering," in Cochlear Prostheses, G. M. Clark, Y. C. Tong and J. F. Patrick, Eds. Edinburgh: Churchill Livingstone, 1990, ch. 6, pp $99-124$

[4] Y. C. Tong, R. C. Black, G. M Clark, I. C. Forster, J. B. Millar, B. J. O'Loughlin, and J. F. Patrick. "A preliminary report on a multiplechannel cochlear implant operation," J. Laryngol. Otol., vol. 93, pp. 679-695, July 1979.

[5] Y. C. Tong, G. M. Clark, P. M. Seligman, and J. F. Patrick, "Speech processing for a multiple-electrode cochlear implant hearing prosthesis," J. Acoust. Soc. Amer., vol. 68, pp. 1897-1899, 1980.

[6] R. C. Dowell, G. M. Clark, P. M. Seligman, and A. M. Brown, "Perception of connected speech without lipreading, using a multichannel hearing prosthesis," Actu Otolaryngol., suppl. 102, Stockholm, Sweden, pp. 7-11, 1986.

[7] P. J. Blamey, R. C. Dowell, A. M. Brown, G. M. Clark, and P M.Seligman, "Vowel and consonant recognition of cochlear implant patients using formant-estimating speech processors," J. Acoust. Soc. Amer., vol. 82, pp. 48-57, 1987.

[8] R. C. Dowell, P. M. Seligman, P. J. Blamey, and G. M. Clark, "Speech perception using a two-formant 22-electrode cochlear prosthesis in quiet," Acta Otolaryngol., suppl. 104, Stockholm, Sweden, pp. 439-446, 1987.

[9] M. W. Skinner, L. K. Holden, T. A. Holden, R. C. Dowell, P. M. Seligman, J. A. Brimacombe, and A. L. Beiter, "Performance of postlinguistically deaf adults with the wearable speech processor (WSP III) and mini speech processor (MSP) of the nucleus multi-electrode cochlear implant," Ear Hear., vol. 12, pp. 3-22, Feb. 1991

[10] H. J. McDermott, C. M. McKay, and A. E. Vandali, "A new portable sound processor for the University of Melbourne/Nucleus Limited multielectrode cochlear implant," J. Acoust. Soc. Amer., vol. 91, pp. 3367-3371, June 1992.

[11] C. M. McKay, H. J. McDermott, A. E. Vandali, and G. M. Clark, "A comparison of speech perception of cochlear implantees using the spectral maxima sound processor (SMSP) and the MSP(MULTIPEAK) processor," Acta Otolaryngol., vol. 112, pp. 752-761, 1992.

[12] C. M. McKay and H. J. McDermott, "Perceptual performance of cochlear implantees using the spectral maxima sound processor (SMSP) and the MSP(MULTIPEAK) processor," Ear Hear., vol. 14, pp 349-367, Oct. 1993.

[13] C. K. Yuen and D. Fraser, Digital Spectral Analysis. Melbourne Australia: CSIRO/Pitman, 1979, pp. 82-83.

[14] W. D. Stanley, G. R. Dougherty, and R. Dougherty, Digital Signal Processing. Reston, 1984, ch. 8, p. 232.

[15] G. Peterson and I. Lehiste, "Revised CNC lists for auditory tests," $J$. Speech Hear. Disorders, vol. 27, pp. 62-70, Feb. 1962

[16] C. M. McKay, A. E. Vandali, H. J. McDermott, and G. M. Clark, "Speech processing for cochlear implants: Variations of the Spectral Maxima Sound Processor," Acta Otolaryngol., to be published.

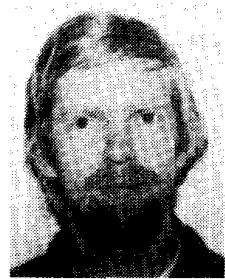

Hugh J. McDermott received the B.App.Sc. degree in electronics from the University of Melbourne, Australia, in 1981. He received the Ph.D. in 1988 for the development of an advanced implantable auditory nerve stimulator.

Since 1982 he has worked in the University of Melbourne on various aspects of cochlear implant and speech processor design. He has obtained a number of patents relating to hearing prosthesis design. Currently, his research interests include the development of improved speech processors for cochlear implants and hearing aids.

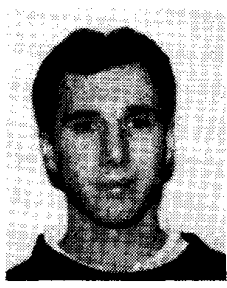

Andrew E. Vandali was born in Melbourne, Australia, on February 7, 1964. He received the B.Eng. degree with Honors in 1985 from the Royal Melbourne Institute of Technology.

He worked for Australian N.C. Automation for four years as an R\&D engineer. In 1989 he joined the Department of Otolaryngology at the University of Melbourne as a Research Engineer working in the field of speech processing for cochlear implants. Since 1992 he has been with the Australian Bionic Ear \& Hearing Research Institute, employed as a Senior Research Engineer, where he has continued work in that field. His areas of research interest include real-time digital signal processing of speech signals, and modeling of cochlear mechanics and neural functions.

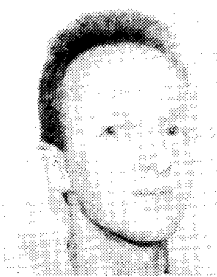

Richard J. M. van Hoesel was born in Limbricht, The Netherlands, on December 23, 1962 He received the B.E. degree in electrical and computer systems engineering at Monash University, Melbourne, Australia, in 1985 . He is currently working towards the completion of a Ph.D. degree at Melbourne University. He has designed real-time DSP systems for computer vision applications with the C.S.I.R.O. Australia, and has been working on the cochlear implant program with the Department of Otolaryngology at the University of Melbourne since 1988. His present research activities are in the areas of binaural hearing and prostheses, real-time DSP applications for cochlear implants, wearable DSP hardware design, and adaptive beam-forming and noise reduction techniques.

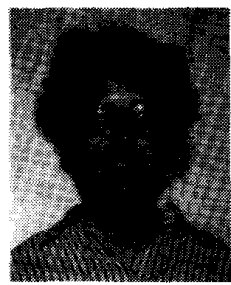

Colette M. McKay received the B.Sc. degree (Hons.) from the University of Melbourne, Australia, in 1974, and received the Ph.D. degree at the same University in 1979 for her thesis in theoretical nuclear physics entitled "The theory of scattering from complex energy dependent potentials, and applications." She then received the Diploma in audiology and has been actively involved in the teaching of audiology up to the present.

Since 1989, she has worked as a Research Fellow in the field of speech processing and psychophysics

for cochlear implants.

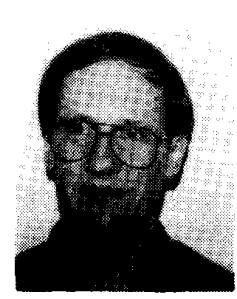

J. Mark Harrison was born in Melbourne, Australia, on March 8, 1957. He received the B.Eng degree in communications from Preston Institute of Technology, Australia, in 1979, and a postgraduate Diploma in digital computer engineering from the Royal Melbourne Institute of Technology in 1984.

Since 1981 he has been employed as a Research Engineer in the Department of Otolaryngology, University of Melbourne and is currently a Senior Research Engineer with the Australian Bionic Ear \& Hearing Research Institute. His main research interests include digital signal processing of speech signals, and hardware development of portable, battery-powered signal processing systems for use with cochlear implants.

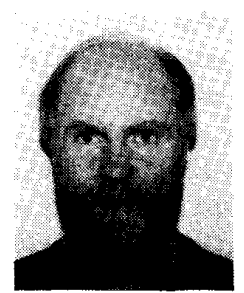

Lawrence T. Cohen received his tertiary education at the University of Melbourne, Australia, originally studying physics, and received the Ph.D. degree in 1990 on the application of steady-state evoked potentials to infant hearing screening.

He has designed and manufactured vacuum tube amplifiers, and has subsequently been involved in cochlear implant research, especially in psychophysics and computer modeling of electrically evoked current spread in the human cochlea. 


\section{University Library}

\section{- M M N E R VA A gateway to Melbourne's research publications}

Minerva Access is the Institutional Repository of The University of Melbourne

Author/s:

McDermott, Hugh J.;Vandali, Andrew E.;van Hoesel, Richard J. M.;McKay, Colette M;HARRISON, MARK;Cohen, Lawrence T.

Title:

A portable programmable digital sound processor for cochlear implant research

Date:

1993

Citation:

McDermott, H. J., Vandali, A. E., van Hoesel, R. J. M., McKay, C. M., Harrison, M., \& Cohen, L. T. (1993). A portable programmable digital sound processor for cochlear implant research. IEEE Transactions on Rehabilitation Engineering, June, 1(2), 94-100.

Persistent Link:

http://hdl.handle.net/11343/27335 